\title{
Epididymitis following Cytoreductive Surgery with Intraperitoneal Oxaliplatin Chemotherapy: Two Case Reports
}

\author{
Luiza Damian Ribeiro Barbosa Marcos Belotto \\ Renata D'Alpino Peixoto \\ Hospital São José, São Paulo, Brazil
}

\section{Key Words}

Epididymitis · Hyperthermic intraperitoneal chemotherapy · Cytoreductive surgery ·

Oxaliplatin

\begin{abstract}
Cytoreductive surgery (CRS) combined with hyperthermic intraperitoneal chemotherapy has emerged as an aggressive treatment option with intent to either cure or extend disease-free survival of selected patients with peritoneal carcinomatosis. However, postoperative complications are common. We describe the cases of 2 men who underwent CRS with hyperthermic intraperitoneal oxaliplatin and developed scrotal pain, which was consistent with noninfectious epididymitis.

(C) 2016 The Author(s)

Published by S. Karger AG, Basel
\end{abstract}

\section{Introduction}

Peritoneal carcinomatosis associated with gastrointestinal malignancies was once considered a very poor prognostic condition $[1,2]$. However, in the last decades, cytoreductive surgery (CRS) combined with hyperthermic intraperitoneal chemotherapy (HIPEC) has gained popularity and emerged as an aggressive treatment option with intent to either cure or extend disease-free survival of selected patients with peritoneal carcinomatosis [3, 4].

This complex multimodal surgical procedure involves extensive stripping of the peritoneal surface and multiple visceral organ resections in order to achieve maximal cytoreduction with no macroscopic residual disease within the abdomen [5]. Following resection of all

\section{KARGER}

Luiza Damian Ribeiro Barbosa Hospital São José

R. Martiniano de Carvalho, 965

São Paulo, SP 01321-001 (Brazil)

E-Mail luizabarb@hotmail.com 
Ribeiro Barbosa et al.: Epididymitis following Cytoreductive Surgery with Intraperitoneal Oxaliplatin Chemotherapy: Two Case Reports

visible tumor, a heated chemotherapy perfusate is administered intraoperatively into the abdominal cavity with the aim of annihilating viable microscopic residual disease. The peritoneal route of chemotherapy is based on the peritoneal-plasma division concept, whereby a tenfold higher concentration of the chemotherapy is reached in the abdominal cavity in direct contact with residual cancerous cells, while minimal systemic absorption and adverse effects are expected.

Despite all those potential benefits, the morbidity and mortality rates associated with CRS and HIPEC are elevated. Large series of patients undergoing CRS with HIPEC report mortality rates varying from 1 to 8\% [6-10]. In the only prospective randomized study, 105 patients were randomly assigned to receive either standard treatment consisting of systemic chemotherapy (fluorouracil-leucovorin) with or without palliative surgery, or experimental therapy consisting of aggressive CRS with HIPEC, followed by the same systemic chemotherapy regime. The treatment-related mortality in CRS with the HIPEC group was 8\% [11]. Surgical morbidity, such as postoperative ileus, anastomotic fistula, and wound infection, as well as medical morbidity, including pharmacological toxicity, cytopenia, bone marrow aplasia, renal toxicity, and hydroelectrolytic disorders are also frequent following CRS with HIPEC $[6,11]$, with a morbidity rate of approximately $25 \%$. The most common described complications include enteric fistula, intraabdominal abscess, pneumonia, small-bowel obstruction, pancreatitis, and neutropenia which often prolong the hospital stay.

To the best of our knowledge, there are no reported cases of epididymitis after CRS with HIPEC. Here, we describe the cases of 2 men who underwent CRS with hyperthermic intraperitoneal oxaliplatin and developed scrotal pain, which was consistent with noninfectious epididymitis.

\section{Case Reports}

Case 1

A 23-year-old man presented in 2011 with increasing abdominal pain. In October 2011, he developed obstructive symptoms and underwent exploratory laparotomy. He was found to have a poorly differentiated adenocarcinoma of the transverse colon with multiple peritoneal implants. It was consistent with a pT4 pN2 (4/28) pM1 adenocarcinoma, K-RAS wild type. From January to July 2012, he received 12 cycles of FOLFIRI plus bevacizumab at another institution and had complete clinical response. Therefore, it was decided to stop his chemotherapy completely. In September 2013, he again developed abdominal pain. A PETCT scan revealed FDG-uptaking lesions in the peritoneum as well as in the colonic anastomosis. From June 2014 to June 2015, he received 24 cycles of FOLFIRI and cetuximab with initial response followed by stable disease. In September 2015, he was first seen at our institution, and during a multidisciplinary meeting, it was decided to pursue CRS with HIPEC (oxaliplatin $300 \mathrm{mg} / \mathrm{m}^{2}$ ), which occurred on October 5, 2015. He stayed at the ICU for only $72 \mathrm{~h}$, and had the first bowel movement 6 days after surgery. On the 7th postoperative day, he developed fever with no identified origin, and was started on piperacillin-tazobactam and vancomycin. At this time, the patient was also complaining of left scrotal pain and swelling. An ultrasound of the testis was performed on October 17th and showed normal testicles and increased volume of the left epididymis with heterogeneous echogenicity. In addition, analysis of the epididymal waveform revealed a low-resistance pattern suggestive of acute epididymitis (fig. 1). Although all cultures remained negative, it was decided to switch antibiotics to amikacin for 10 days and doxycycline for 14 days and treat as infectious epididymitis. The patient was discharged home on October 22, 2015, to complete antibiotics at the outpatient 
Ribeiro Barbosa et al.: Epididymitis following Cytoreductive Surgery with Intraperitoneal Oxaliplatin Chemotherapy: Two Case Reports

unit. As of November 23, the patient was still facing left scrotal discomfort, although less in intensity.

Case 2

A 57-year-old man presented in August 2014 with epigastralgia. An upper endoscopy was requested and revealed a $3-\mathrm{cm}$ infiltrative ulcer in the posterior wall of the stomach. Biopsy confirmed a well-differentiated adenocarcinoma, HER-2 3+. A staging CT scan showed peritoneal carcinomatosis, and the CEA was $256 \mathrm{ng} / \mathrm{ml}$. In October 2014, he was started on FLOT plus trastuzumab and achieved an excellent response. In June 2015, his CEA was 2.2 , and he was completely asymptomatic. On August 17, 2015, the patient underwent CRS with hyperthermic intraperitoneal oxaliplatin at a dose of $300 \mathrm{mg} / \mathrm{m}^{2}$ as well as gastrectomy and lymphadenectomy. The patient stayed at the ICU for $72 \mathrm{~h}$ and had no major adverse event. He had a bowel movement 5 days after surgery. His only complaint was fatigue and moderate right scrotal pain with no inflammatory signs. The scrotal pain subsequently improved over the course of 3 months.

\section{Discussion}

CRS with HIPEC has proven to be an effective treatment modality for selected patients with peritoneal surface malignancies. However, postoperative complications are very common, such as anastomotic leakage, intestinal obstruction, and pancreatitis [12]. To the best of our knowledge, no previous reports exist in the literature regarding epididymitis following CRS with HIPEC. Both of our case reports describe men who underwent CRS with hyperthermic intraperitoneal oxaliplatin and developed scrotal pain, which was consistent with noninfectious epididymitis.

Epididymitis is the most common cause of scrotal pain in adults [13], and its most common etiology is infectious, although it can also be due to noninfectious causes, such as trauma and autoimmune disease [14]. Noninfectious epididymitis is generally a chronic condition. Other noninfectious causes of epididymitis include vasculitis and certain medications, such as amiodarone [15]. However, no etiology is found in many cases.

Risk factors for epididymitis in all men include sexual activity, strenuous physical activity, bicycle or motorcycle riding, and prolonged periods of sitting (e.g., during travel, with a sedentary job) [13]. Risk factors in men older than 35 years and in prepubertal boys include recent urinary tract surgery or instrumentation and anatomic abnormalities, such as prostatic obstruction in older men and posterior urethral valves or meatal stenosis in prepubertal boys $[13,14]$. Our patients had none of the risk factors described previously.

Unlike patients with infectious epididymitis, patients with noninfectious epididymitis have less epididymal inflammation, such as pain and swelling [13-16]. The diagnosis is usually made after the exclusion of other etiologies, particularly infectious epididymitis. No further evaluation is necessary for noninfectious epididymitis [14]. In the first case, signs and symptoms were more intense, and the ultrasound of the testis was helpful in defining the diagnosis. In the second case, the pain was only mild and no imaging tests were requested. Interestingly, in both cases pain was still present a few months after surgery, suggesting a more chronic condition.

The physiopathology behind noninfectious epididymitis following CRS with HIPEC remains unknown. Usually, the testes descend spontaneously into the scrotum by 4 months of age. The mechanisms responsible for normal testicular descent are not well understood. The intraabdominal phase of descent is thought to be androgen-independent. Passage through 
Ribeiro Barbosa et al.: Epididymitis following Cytoreductive Surgery with Intraperitoneal Oxaliplatin Chemotherapy: Two Case Reports

the inguinal canal, which begins in the 28th week of gestation, is believed to result from interaction between mechanical, hormonal, and neurotransmitter effects [17]. One of our hypotheses for epididymitis following HIPEC is that maybe both patients had patency of the processus vaginalis. Therefore, during intraperitoneal oxaliplatin administration, a small amount of oxaliplatin could have gained access to the scrotum through the processus vaginalis, leading to testis inflammation and epididymitis.

It remains unknown whether other intraperitoneal chemotherapeutic agents could also cause epididymitis or it is an oxaliplatin-related effect. Also, we have not found any report in the literature regarding epididymitis as a side effect of intravenous oxaliplatin, which is very frequently used in clinical practice. In conclusion, although rare, epididymitis should be noted as one of the potential complications following CRS with intraperitoneal oxaliplatin.

\section{Statement of Ethics}

The corresponding author acknowledges that she is responsible for complying with ethical requirements and declares that the patients were correctly informed and written informed consent was obtained; the confidentiality of the patients was strictly preserved; the patients were informed about the submission of the manuscript and will be acquainted when the article is published.

\section{Disclosure Statement}

The authors declare that they have nothing to disclose.

\section{References}

1 Lang NP, Thompson C, Chu DZ, et al: Peritoneal carcinomatosis in nongynecologic malignancy. A prospective study of prognostic factors. Cancer 1989;63:364-367.

2 Sadeghi B, Arvieux C, Glehen 0, et al: Carcinomatosis from non-gynecologic malignancies: results of the EVOCAPE-1 multicentric prospective study. Cancer 2000;88:358-363.

$\$ 3$ Levine EA, Stewart JH, Russell GB, et al: Cytoreductive surgery and intraperitoneal hyperthermic chemotherapy for peritoneal surface malignancy: experience with 501 procedures. J Am Coll Surg 2007;204:943-953.

-4 Sugarbaker PH, Cunliffe WJ, Belliveau J, et al: Rationale for integrating early postoperative intraperitoneal chemotherapy into the surgical treatment of gastrointestinal cancer. Semin Oncol 1989;16(suppl 6):83-97.

5 Verwaal VJ, Bruin S, Boot H: 8-year follow-up of randomized trial: cytoreduction and hyperthermic intraperitoneal chemotherapy versus systemic chemotherapy in patients with peritoneal carcinomatosis of colorectal cancer. Ann Surg Oncol 2008;15:754-763.

-6 Stephens AD, Alderman R, Chang D, et al: Morbidity and mortality analysis of 200 treatments with cytoreductive surgery and hyperthermic intraoperative intraperitoneal chemotherapy using the coliseum technique. Ann Surg Oncol 1999;6:790-796.

7 Glehen 0, Osinsky D, Cotte E, et al: Intraperitoneal chemohyperthermia using a closed abdominal procedure and cytoreductive surgery for the treatment of peritoneal carcinomatosis: morbidity and mortality analysis of 216 consecutive procedures. Ann Surg Oncol 2003;10:863-869.

-8 Kusamura S, Younan R, Baratti D, et al: Cytoreductive surgery followed by intraperitoneal hyperthermic perfusion. Analysis of morbidity and mortality in 209 peritoneal surface malignancies treated with closed abdomen technique. Cancer 2006;106:1144-1153.

-9 Nadler A, McCart JA, Govindarajan A: Peritoneal carcinomatosis from colon cancer: a systematic review of the data for cytoreduction and intraperitoneal chemotherapy. Clin Colon Rectal Surg 2015;28:234-246.

10 Desantis M, Bernard JL, Casanova V, et al: Morbidity, mortality, and oncological outcomes of 401 consecutive cytoreductive procedures with hyperthermic intraperitoneal chemotherapy (HIPEC). Langenbecks Arch Surg 2015;400:37-48. 


\section{Case Reports in Oncology}

\begin{tabular}{l|l}
\hline Case Rep Oncol 2016;9:138-142 \\
\hline DOI: $10.1159 / 000443726$ & $\begin{array}{l}\text { (c) 2016 The Author(s). Published by S. Karger AG, Basel } \\
\text { www.karger.com/cro }\end{array}$ \\
\hline
\end{tabular}

Ribeiro Barbosa et al.: Epididymitis following Cytoreductive Surgery with Intraperitoneal Oxaliplatin Chemotherapy: Two Case Reports

11 Verwaal VJ, Ruth SV, Bree E, et al: Randomized trial of cytoreduction and hyperthermic intraperitoneal chemotherapy versus systemic chemotherapy and palliative surgery in patients with peritoneal carcinomatosis of colorectal cancer. J Clin Oncol 2003;21:3737-3743.

12 Lampl B: Leebmann H, Mayr M, et al: Rare diaphragmatic complications following cytoreductive surgery and HIPEC: report of two cases. Surg Today 2014;44:383-386.

13 Trojian TH, Lishnak TS, Heiman D: Epididymitis and orchitis: an overview. Am Fam Physician 2009;79:583.

14 Tracy CR, Steers WD, Costabile R: Diagnosis and management of epididymitis. Urol Clin North Am 2008;35:101.

15 Nikolaou M, Ikonomidis I, Lekakis I, et al: Amiodarone-induced epididymitis: a case report and review of the literature. Int J Cardiol 2007;121:e15-e16.

16 Walker NA, Challacombe B: Managing epididymo-orchitis in general practice. Practitioner 2013;257:21-25, 2-3.

17 Wensing CJ: The embryology of testicular descent. Horm Res 1988;30:144.

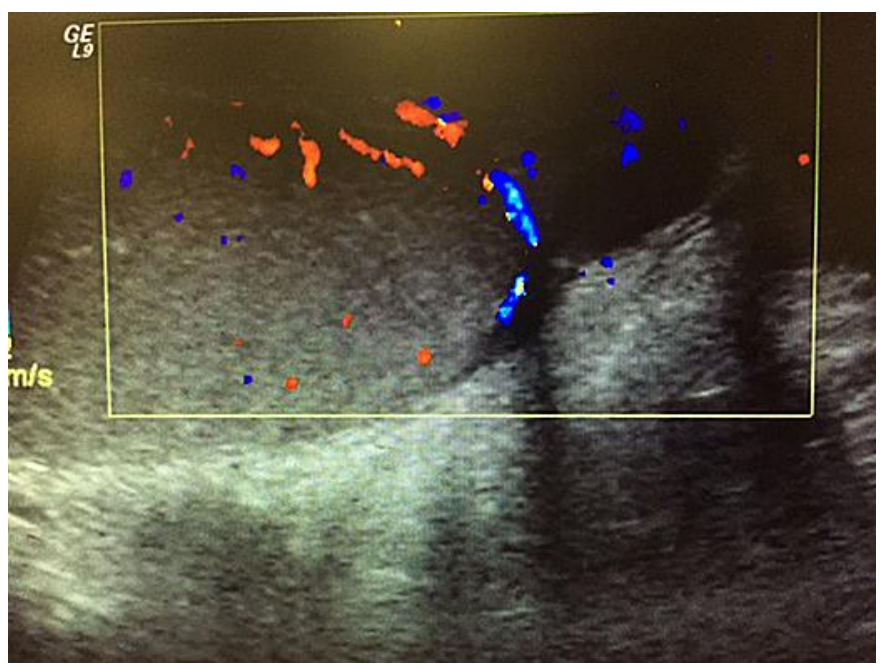

Fig. 1. Ultrasound showing increased volume of the left epididymis with heterogeneous echogenicity. 